\title{
Non-hermitian extensions of Heisenberg type and Schrödinger type uncertainty relations
}

\author{
Kenjiro Yanagi* and Kohei Sekikawa
}

"Correspondence:

yanagi@yamaguchi-u.ac.jp

Graduate School of Science and

Engineering, Yamaguchi University,

Ube, 755-8611, Japan

\begin{abstract}
In quantum mechanics it is well known that the Heisenberg-Schrödinger uncertainty relations hold for two non-commutative observables and density operator. Recently Dou and Du (J. Math. Phys. 54:103508, 2013; Int. J. Theor. Phys. 53:952-958, 2014) obtained several uncertainty relations for two non-commutative non-hermitian observables and density operators. In this paper, we show that their results can be given as corollaries of our non-hermitian extensions of Heisenberg type or Schrödinger type uncertainty relations for the generalized metric adjusted skew information or generalized metric adjusted correlation measures which were obtained in Furuichi and Yanagi (J. Math. Anal. Appl. 388:1147-1156, 2012).
\end{abstract}

MSC: Primary 15A45; 47A63; secondary 94A17

Keywords: trace inequality; metric adjusted skew information; non-hermitian observable

\section{Introduction}

Let $M_{n}(\mathbb{C})\left(\right.$ resp. $\left.M_{n, s a}(\mathbb{C})\right)$ be the set of all $n \times n$ complex matrices (resp. all $n \times n$ selfadjoint matrices), endowed with the Hilbert-Schmidt scalar product $\langle A, B\rangle=\operatorname{Tr}\left[A^{*} B\right]$. Let $M_{n,+}(\mathbb{C})$ be the set of strictly positive elements of $M_{n}(\mathbb{C})$ and $M_{n,+, 1}(\mathbb{C}) \subset M_{n,+}(\mathbb{C})$ be the set of strictly positive density matrices, that is, $M_{n,+1}(\mathbb{C})=\left\{\rho \in M_{n}(\mathbb{C}) \mid \operatorname{Tr}[\rho]=1, \rho>0\right\}$. If it is not otherwise specified, from now on we shall treat the case of faithful states, that is, $\rho>0$. It is well known that the expectation of an observable $A \in M_{n, s a}(\mathbb{C})$ in a state $\rho \in M_{n,+, 1}(\mathbb{C})$ is defined by

$$
E_{\rho}(A):=\operatorname{Tr}(\rho A)
$$

and the variance of an observable $A \in M_{n, s a}(\mathbb{C})$ in a state $\rho \in M_{n,+, 1}(\mathbb{C})$ is defined by

$$
V_{\rho}(A)=\operatorname{Tr}\left[\rho\left(A-E_{\rho}(A) I\right)^{2}\right] .
$$

In order to represent the degree of non-commutativity between $\rho \in M_{n,+, 1}(\mathbb{C})$ and $A \in$ $M_{n, s a}(\mathbb{C})$, the Wigner-Yanase skew information $I_{\rho}(A)$ is defined by

$$
I_{\rho}(A)=\frac{1}{2} \operatorname{Tr}\left[\left(i\left[\rho^{1 / 2}, A\right]\right)^{2}\right]=\operatorname{Tr}\left[\rho A^{2}\right]-\operatorname{Tr}\left[\rho^{1 / 2} A \rho^{1 / 2} A\right],
$$

(C) 2015 Yanagi and Sekikawa. This article is distributed under the terms of the Creative Commons Attribution 4.0 International License (http://creativecommons.org/licenses/by/4.0/), which permits unrestricted use, distribution, and reproduction in any medium, provided you give appropriate credit to the original author(s) and the source, provide a link to the Creative Commons license, and indicate if changes were made. 
where $[X, Y]=X Y-Y X$. Furthermore the Wigner-Yanase-Dyson skew information $I_{\rho, \alpha}(A)$ is defined by

$$
I_{\rho, \alpha}(A)=\frac{1}{2} \operatorname{Tr}\left[\left(i\left[\rho^{\alpha}, A\right]\right)\left(i\left[\rho^{1-\alpha}, A\right]\right)\right]=\operatorname{Tr}\left[\rho A^{2}\right]-\operatorname{Tr}\left[\rho^{\alpha} A \rho^{1-\alpha} A\right] \quad(\alpha \in[0,1]) .
$$

The convexity of $I_{\rho, \alpha}(A)$ with respect to $\rho$ was famously shown by Lieb [4]. The relationship between Wigner-Yanase skew information and the uncertainty relation was given by Luo and Zhang [5] for the first time. Afterward, the relationship between Wigner-YanaseDyson skew information and the uncertainty relation was given by Kosaki [6] and Yanagi $e t$ $a l$. [7]. Furthermore metric adjusted skew information was defined by Hansen [8] which is an extension of Wigner-Yanase-Dyson skew information. The relationship between metric adjusted skew information and the uncertainty relation was given by Yanagi [9] and was generalized in Yanagi et al. [10] for generalized metric adjusted skew information and generalized metric adjusted correlation measures. In this paper we give some non-hermitian extensions of Heisenberg type and Schrödinger type uncertainty relations related to generalized quasi-metric adjusted skew information and generalized quasi-metric adjusted correlation measures. As a result we can obtain some results of non-hermitian uncertainty relations given by Dou and $\mathrm{Du}$ as corollaries of our results.

\section{Dou-Du's non-hermitian uncertainty relations}

Definition 1 For $A, B \in M_{n}(\mathbb{C}), \rho \in M_{n,+, 1}(\mathbb{C})$, we define the following.

(1) $[A, B]^{0}=\frac{1}{2}\left([A, B]+\left[A^{*}, B^{*}\right]\right)$, where $[A, B]=A B-B A$.

(2) $\{A, B\}^{0}=\frac{1}{2}\left(\{A, B\}+\left\{A^{*}, B^{*}\right\}\right)$, where $\{A, B\}=A B+B A$.

(3) $\left|\operatorname{Var}_{\rho}\right|(A)=\operatorname{Tr}\left[\rho A_{0}^{*} A_{0}\right]$, where $A_{0}=A-\operatorname{Tr}[\rho A] I$.

(4) $\left|\operatorname{Var}_{\rho}^{0}\right|(A)=\frac{1}{2}\left(\left|\operatorname{Var}_{\rho}\right|(A)+\left|\operatorname{Var}_{\rho}\right|\left(A^{*}\right)\right)$.

Theorem 1 (Dou-Du [2]) For $A, B \in M_{n}(\mathbb{C}), \rho \in M_{n,+1}(\mathbb{C})$, we have the following.

(1) $\left|\operatorname{Var}_{\rho}^{0}\right|(A)\left|\operatorname{Var}_{\rho}^{0}\right|(B) \geq \frac{1}{4}|\operatorname{Tr}[\rho[A, B]]|^{2}$.

(2) $\left|\operatorname{Var}_{\rho}^{0}\right|(A)\left|\operatorname{Var}_{\rho}^{0}\right|(B) \geq \frac{1}{4}\left|\operatorname{Tr}\left[\rho\left\{A_{0}, B_{0}\right\}\right]\right|^{2}$.

(3) $\left|\operatorname{Var}_{\rho}^{0}\right|(A)\left|\operatorname{Var}_{\rho}^{0}\right|(B) \geq \frac{1}{4}\left|\operatorname{Tr}\left[\rho[A, B]^{0}\right]\right|^{2}+\frac{1}{4}\left|\operatorname{Tr}\left[\rho\left\{A_{0}, B_{0}\right\}^{0}\right]\right|^{2}$.

(4) $\left|U_{\rho}\right|(A)\left|U_{\rho}\right|(B) \geq \frac{1}{4}\left|\operatorname{Tr}\left[\rho[A, B]^{0}\right]\right|^{2}$, where

$$
\begin{aligned}
& \left|U_{\rho}\right|(A)=\sqrt{\left(\left|\operatorname{Var}_{\rho}^{0}\right|(A)\right)^{2}-\left(\left|\operatorname{Var}_{\rho}^{0}\right|(A)-\left|I_{\rho}\right|(A)\right)^{2}}, \\
& \left|I_{\rho}\right|(A)=\frac{1}{2} \operatorname{Tr}\left[\left(i\left[\rho^{1 / 2}, A^{*}\right]\right)\left(i\left[\rho^{1 / 2}, A\right]\right)\right] .
\end{aligned}
$$

\section{Quantum Fisher information}

A function $f:(0,+\infty) \rightarrow \mathbb{R}$ is said operator monotone if, for any $n \in \mathbb{N}$, and $A, B \in M_{n,+}(\mathbb{C})$ such that $0 \leq A \leq B$, the inequalities $0 \leq f(A) \leq f(B)$ hold. An operator monotone function is said symmetric if $f(x)=x f\left(x^{-1}\right)$ and normalized if $f(1)=1$. The following definitions were given by Hansen and Gibilisco, etc.

Definition $2 \mathcal{F}_{\text {op }}$ is the class of functions $f:(0,+\infty) \rightarrow(0,+\infty)$ satisfying

(1) $f(1)=1$,

(2) $t f\left(t^{-1}\right)=f(t)$,

(3) $f$ is operator monotone. 
For $f \in \mathcal{F}_{\text {op }}$ define $f(0)=\lim _{x \rightarrow 0} f(x)$. We introduce the sets of regular and non-regular functions

$$
\mathcal{F}_{\text {op }}^{r}=\left\{f \in \mathcal{F}_{\text {op }} \mid f(0) \neq 0\right\}, \quad \mathcal{F}_{\text {op }}^{n}=\left\{f \in \mathcal{F}_{\text {op }} \mid f(0)=0\right\}
$$

and notice that trivially $\mathcal{F}_{\text {op }}=\mathcal{F}_{\text {op }}^{r} \cup \mathcal{F}_{\text {op }}^{n}$.

Definition 3 For $f \in \mathcal{F}_{\mathrm{op}}^{r}$ we define

$$
\tilde{f}(x)=\frac{1}{2}\left[(x+1)-(x-1)^{2} \frac{f(0)}{f(x)}\right], \quad x>0 .
$$

Proposition 1 ([11]) The correspondence $f \rightarrow \tilde{f}$ is a bijection between $\mathcal{F}_{\mathrm{op}}^{r}$ and $\mathcal{F}_{\mathrm{op}}^{n}$.

\section{Example 1}

$$
\begin{aligned}
& f_{R L D}(x)=\frac{2 x}{x+1}, \quad f_{B K N}(x)=\frac{x-1}{\log x} \\
& f_{S L D}(x)=\frac{x+1}{2}, \quad \tilde{f}_{S L D}(x)=\frac{2 x}{x+1} \\
& f_{W Y}(x)=\left(\frac{\sqrt{x}+1}{2}\right)^{2}, \quad \tilde{f}_{W Y}(x)=\sqrt{x} \\
& f_{W Y D}(x)=\alpha(1-\alpha) \frac{(x-1)^{2}}{\left(x^{\alpha}-1\right)\left(x^{1-\alpha}-1\right)}, \quad \alpha \in(0,1), \\
& \tilde{f}_{W Y D}(x)=\frac{1}{2}\left\{x+1-\left(x^{\alpha}-1\right)\left(x^{1-\alpha}-1\right)\right\}, \\
& \frac{2 x}{x+1}<\sqrt{x}<\frac{x-1}{\log x}<f_{W Y D}<\left(\frac{\sqrt{x}+1}{2}\right)^{2}<\frac{x+1}{2} \quad(x \neq 1) .
\end{aligned}
$$

In the Kubo-Ando theory of matrix means one associates a mean to each operator monotone function $f \in \mathcal{F}_{\text {op }}$ by the formula

$$
m_{f}(A, B)=A^{1 / 2} f\left(A^{-1 / 2} B A^{-1 / 2}\right) A^{1 / 2},
$$

where $A, B \in M_{n,+}(\mathbb{C})$.

By using the notion of matrix means one may define the class of monotone metrics (also called quantum Fisher information) by the following formula:

$$
\langle A, B\rangle_{\rho, f}=\operatorname{Tr}\left[A \cdot m_{f}\left(L_{\rho}, R_{\rho}\right)^{-1}(B)\right],
$$

where $L_{\rho}(A)=\rho A, R_{\rho}(A)=A \rho$.

\section{Generalized quasi-metric adjusted skew information and correlation}

measure

Definition 4 Let $g, f \in \mathcal{F}_{\text {op }}^{r}$ satisfy

$$
g(x) \geq k \frac{(x-1)^{2}}{f(x)}
$$


for some $k>0$. We define

$$
\Delta_{g}^{f}(x)=g(x)-k \frac{(x-1)^{2}}{f(x)} \in \mathcal{F}_{\mathrm{op}}
$$

By Lemma 5.2 in [12], $-k \frac{(x-1)^{2}}{f(x)}$ is operator concave. Then $\Delta_{g}^{f}(x)$ is also operator concave. Since $\Delta_{g}^{f}(x)>0$ for $(0, \infty), \Delta_{g}^{f}(x)$ is operator monotone.

Definition 5 For $A, B \in M_{n}(\mathbb{C})$ and $\rho \in M_{n,+1}(\mathbb{C})$, we define the following quantities:

$$
\begin{aligned}
& \left|\operatorname{Corr}_{\rho}^{(g, f)}\right|(A, B)=k\langle i[\rho, A], i[\rho, B]\rangle_{\rho, f}, \\
& \left|I_{\rho}^{(g, f)}\right|(A)=\left|\operatorname{Corr}_{\rho}^{(g, f)}\right|(A, A), \\
& \left|C_{\rho}^{f}\right|(A, B)=\operatorname{Tr}\left[A^{*} m_{f}\left(L_{\rho}, R_{\rho}\right) B\right], \quad\left|C_{\rho}^{f}\right|(A)=\left|C_{\rho}^{f}\right|(A, A), \\
& \left|U_{\rho}^{(g, f)}\right|(A)=\sqrt{\left(\left|C_{\rho}^{g}\right|(A)+\left|C_{\rho}^{\Delta_{g}^{f}}\right|(A)\right)\left(\left|C_{\rho}^{g}\right|(A)-\left|C_{\rho}^{\Delta_{g}^{f}}\right|(A)\right) .}
\end{aligned}
$$

The quantity $\left|I_{\rho}^{(g, f)}\right|(A)$ and $\left|\operatorname{Corr}_{\rho}^{(g, f)}\right|(A, B)$ are called generalized quasi-metric adjusted skew information and generalized quasi-metric adjusted correlation measures, respectively.

Then we have the following proposition.

Proposition 2 For $A, B \in M_{n}(\mathbb{C})$ and $\rho \in M_{n,+1}(\mathbb{C})$, we have the following relations:

(1) $\left|I_{\rho}^{(g, f)}\right|(A)=\left|I_{\rho}^{(g, f)}\right|\left(A_{0}\right)=\left|C_{\rho}^{g}\right|\left(A_{0}\right)-\left|C_{\rho}^{\Delta_{g}^{f}}\right|\left(A_{0}\right)$,

(2) $\left|J_{\rho}^{(g, f)}\right|(A)=\left|C_{\rho}^{g}\right|\left(A_{0}\right)+\left|C_{\rho}^{\Delta_{g}^{f}}\right|\left(A_{0}\right)$,

(3) $\left|U_{\rho}^{(g, f)}\right|(A)=\sqrt{\left|I_{\rho}^{(g, f)}\right|(A) \cdot\left|J_{\rho}^{(g, f)}\right|(A)}$,

(4) $\left|\operatorname{Corr}_{\rho}^{(g, f)}\right|(A, B)=\left|\operatorname{Corr}_{\rho}^{(g, f)}\right|\left(A_{0}, B_{0}\right)$, where $A_{0}=A-\operatorname{Tr}[\rho A] I$ and $B_{0}=B-\operatorname{Tr}[\rho B] I$.

Theorem 2 (Schrödinger type) For $f \in \mathcal{F}_{\text {op }}^{r}$, we have

$$
\left|I_{\rho}^{(g, f)}\right|(A) \cdot\left|I_{\rho}^{(g, f)}\right|(B) \geq|| \operatorname{Corr}_{\rho}^{(g, f)}|(A, B)|^{2},
$$

where $A, B \in M_{n}(\mathbb{C})$ and $\rho \in M_{n,+1}(\mathbb{C})$.

Proof of Theorem 1 By Schwarz's inequality we have

$$
\langle A, A\rangle_{\rho_{f} f}\langle B, B\rangle_{\rho_{f} f} \geq\left|\langle A, B\rangle_{\rho_{f} f}\right|^{2}
$$

Then we have

$$
\begin{aligned}
& \left|I_{\rho}^{(g, f)}\right|(A) \cdot\left|I_{\rho}^{(g, f)}\right|(B) \\
& \quad=\left|\operatorname{Corr}_{\rho}^{(g, f)}\right|(A, A) \cdot\left|\operatorname{Corr}_{\rho}^{(g, f)}\right|(B, B) \\
& \quad \geq|| \operatorname{Corr}_{\rho}^{(g, f)}|(A, B)|^{2} .
\end{aligned}
$$


Theorem 3 (Heisenberg type) For $f \in \mathcal{F}_{\text {op }}^{r}$, if

$$
g(x)+\Delta_{g}^{f}(x) \geq \ell f(x)
$$

for some $\ell>0$, then we have

$$
\left|U_{\rho}^{(g, f)}\right|(A) \cdot\left|U_{\rho}^{(g, f)}\right|(B) \geq k \ell|\operatorname{Tr}(\rho[A, B])|^{2},
$$

where $A, B \in M_{n}(\mathbb{C})$ and $\rho \in M_{n,+, 1}(\mathbb{C})$.

We need the following lemma in order to prove Theorem 3.

Lemma 1 If (1) and (2) is satisfied, then

$$
m_{g}(x, y)^{2}-m_{\Delta_{g}^{f}}(x, y)^{2} \geq k \ell(x-y)^{2} .
$$

Proof By (1) and (2),

$$
\begin{aligned}
& m_{\Delta_{g}^{f}}(x, y)=m_{g}(x, y)-k \frac{(x-y)^{2}}{m_{f}(x, y)}, \\
& m_{g}(x, y)+m_{\Delta_{g}^{f}}(x, y) \geq \ell m_{f}(x, y) .
\end{aligned}
$$

Then by (4) and (5), we have

$$
\begin{aligned}
& m_{g}(x, y)^{2}-m_{\Delta_{g}^{f}}(x, y)^{2} \\
& \quad=\left\{m_{g}(x, y)-m_{\Delta_{g}^{f}}(x, y)\right\}\left\{m_{g}(x, y)+m_{\Delta_{g}^{f}}(x, y)\right\} \\
& \quad \geq \frac{k(x-y)^{2}}{m_{f}(x, y)} \ell m_{f}(x, y) \\
& =k \ell(x-y)^{2} .
\end{aligned}
$$

Proof of Theorem 2 Let $\left\{\left|\phi_{1}\right\rangle,\left|\phi_{2}\right\rangle, \ldots,\left|\phi_{n}\right\rangle\right\}$ be a basis of eigenvectors of $\rho$, corresponding to the eigenvalues $\left\{\lambda_{1}, \lambda_{2}, \ldots, \lambda_{n}\right\}$. We put $a_{j k}=\left\langle\phi_{j}\left|A_{0}\right| \phi_{k}\right\rangle, b_{j k}=\left\langle\phi_{j}\left|B_{0}\right| \phi_{k}\right\rangle$. Then we have

$$
\begin{aligned}
& \left|I_{\rho}^{(g, f)}\right|(A) \\
& =\left|C_{\rho}^{g}\right|\left(A_{0}\right)-\left|C_{\rho}^{\Delta_{g}^{f}}\right|\left(A_{0}\right) \\
& =\operatorname{Tr}\left[A_{0}^{*} m_{f}\left(L_{\rho}, R_{\rho}\right) A_{0}\right]-\operatorname{Tr}\left[A_{0}^{*} m_{\Delta_{g}^{f}}\left(L_{\rho}, R_{\rho}\right) A_{0}\right] \\
& =\sum_{i, j}\left\{m_{f}\left(\lambda_{i}, \lambda_{j}\right)-m_{\Delta_{g}^{f}}\left(\lambda_{i}, \lambda_{j}\right)\right\}\left|a_{i j}\right|^{2}
\end{aligned}
$$

and

$$
\left|J_{\rho}^{(g, f)}\right|(A)=\sum_{i, j}\left\{m_{f}\left(\lambda_{i}, \lambda_{j}\right)+m_{\Delta_{g}^{f}}\left(\lambda_{i}, \lambda_{j}\right)\right\}\left|a_{i j}\right|^{2} .
$$


We remark that

$$
\operatorname{Tr}(\rho[A, B])=\sum_{i} \sum_{j}\left(\lambda_{i}-\lambda_{j}\right) a_{i j} b_{j i}
$$

and

$$
|\operatorname{Tr}(\rho[A, B])| \leq \sum_{i} \sum_{j}\left|\lambda_{i}-\lambda_{j}\right|\left|a_{i j}\right|\left|b_{j i}\right| .
$$

By Lemma 1 we have

$$
\begin{aligned}
k \ell & \left.\operatorname{Tr}[\rho[A, B]]\right|^{2} \\
& \leq\left\{\sum_{i} \sum_{j} \sqrt{k l}\left|\lambda_{i}-\lambda_{j}\right|\left|a_{i j}\right|\left|b_{j i}\right|\right\}^{2} \\
& \leq\left\{\sum_{i} \sum_{j}\left(m_{g}\left(\lambda_{i}, \lambda_{j}\right)^{2}-m_{\Delta_{g}^{f}}\left(\lambda_{i}, \lambda_{j}\right)^{2}\right)^{1 / 2}\left|a_{i j}\right|\left|b_{j i}\right|\right\}^{2} \\
& \leq\left\{\sum_{i} \sum_{j}\left(m_{g}\left(\lambda_{i}, \lambda_{j}\right)-m_{\Delta_{g}^{f}}\left(\lambda_{i}, \lambda_{j}\right)\right)\left|a_{i j}\right|^{2}\right\}\left\{\sum_{i} \sum_{j}\left(m_{g}\left(\lambda_{i}, \lambda_{j}\right)+m_{\Delta_{g}^{f}}\left(\lambda_{i}, \lambda_{j}\right)\right)\left|b_{j i}\right|^{2}\right\} \\
& =I_{\rho}^{(g, f)}(A) J_{\rho}^{(g, f)}(B) .
\end{aligned}
$$

Similarly we have

$$
k \ell|\operatorname{Tr}[\rho[A, B]]|^{2} \leq I_{\rho}^{(g, f)}(B) J_{\rho}^{(g, f)}(A) .
$$

Therefore

$$
\left|U_{\rho}^{(g, f)}\right|(A) \cdot\left|U_{\rho}^{(g, f)}\right|(B) \geq k \ell|\operatorname{Tr}(\rho[A, B])|^{2} .
$$

Example 2 When

$$
\begin{aligned}
& g(x)=\frac{x+1}{2}, \quad f(x)=\alpha(1-\alpha) \frac{(x-1)^{2}}{\left(x^{\alpha}-1\right)\left(x^{1-\alpha}-1\right)} \quad(0<\alpha<1), \\
& k=\frac{f(0)}{2}, \quad \ell=2,
\end{aligned}
$$

we can show positivity:

$$
\Delta_{g}^{f}(x)=g(x)-k \frac{(x-1)^{2}}{f(x)}=\frac{1}{2}\left(x^{\alpha}+x^{1-\alpha}\right) \geq 0
$$

and

$$
\begin{aligned}
g(x)+\Delta_{g}^{f}(x)-\ell f(x) & =\frac{1}{2\left(x^{\alpha}-1\right)\left(x^{1-\alpha}-1\right)}\left\{\left(x^{2 \alpha}-1\right)\left(x^{2(1-\alpha)}-1\right)-4 \alpha(1-\alpha)(x-1)^{2}\right\} \\
& \geq 0 .
\end{aligned}
$$


Then

$$
\begin{aligned}
\left|I_{\rho}^{(f, g)}\right|(A)= & \left|I_{\rho}^{(f, g)}\right|\left(A_{0}\right)=\frac{1}{2} \operatorname{Tr}\left[\rho A_{0} A_{0}^{*}\right]+\frac{1}{2} \operatorname{Tr}\left[\rho A_{0}^{*} A_{0}\right] \\
& -\frac{1}{2} \operatorname{Tr}\left[\rho^{\alpha} A_{0} \rho^{1-\alpha} A_{0}^{*}\right]-\frac{1}{2} \operatorname{Tr}\left[\rho^{1-\alpha} A_{0}^{*} \rho^{\alpha} A_{0}\right] .
\end{aligned}
$$

In particular, for $\alpha=1 / 2$,

$$
\left|I_{\rho}^{(f, g)}\right|(A)=\left|I_{\rho}^{(f, g)}\right|\left(A_{0}\right)=\frac{1}{2} \operatorname{Tr}\left[\rho A_{0} A_{0}^{*}\right]+\frac{1}{2} \operatorname{Tr}\left[\rho A_{0}^{*} A_{0}\right]-\operatorname{Tr}\left[\rho^{1 / 2} A_{0} \rho^{1 / 2} A_{0}^{*}\right] .
$$

In this case we can give some results by Dou-Du as corollaries.

Corollary 1 (Dou-Du (4)) For $A, B \in M_{n}(\mathbb{C})$ and $\rho \in M_{n,+, 1}(\mathbb{C})$,

$$
\begin{aligned}
& \left|U_{\rho}\right|(A) \cdot\left|U_{\rho}\right|(B) \\
& \quad \geq \frac{1}{4}|\operatorname{Tr}[\rho[A, B]]|^{2} \geq \frac{1}{4}|\operatorname{Im} \operatorname{Tr}[\rho[A, B]]|^{2} \\
& \quad=\frac{1}{4}\left|\frac{1}{2} \operatorname{Tr}[\rho[A, B]]-\frac{1}{2} \operatorname{Tr}[\rho[A, B]]\right|^{2} \\
& \quad=\frac{1}{4}\left|\frac{1}{2} \operatorname{Tr}[\rho[A, B]]+\frac{1}{2} \operatorname{Tr}\left[\rho\left[A^{*}, B^{*}\right]\right]\right|^{2} \\
& \quad=\frac{1}{4}\left|\operatorname{Tr}\left[\rho \frac{[A, B]+\left[A^{*}, B^{*}\right]}{2}\right]\right|^{2} \\
& \quad=\frac{1}{4}\left|\operatorname{Tr}\left[\rho[A, B]^{0}\right]\right|^{2} .
\end{aligned}
$$

Corollary 2 (Dou-Du (1), (2)) For $A, B \in M_{n}(\mathbb{C})$ and $\rho \in M_{n,+, 1}(\mathbb{C})$,

(1) $\left|V_{\rho}\right|(A) \cdot\left|V_{\rho}\right|(B) \geq\left|U_{\rho}\right|(A) \cdot\left|U_{\rho}\right|(B) \geq \frac{1}{4}|\operatorname{Tr}[\rho[A, B]]|^{2}$.

(2) $\left|V_{\rho}\right|(A) \cdot\left|V_{\rho}\right|(B) \geq \frac{1}{4}\left|\operatorname{Tr}\left[\rho\left\{A_{0}, B_{0}\right\}\right]\right|^{2}$.

Proof

(1) It is clear by Theorem 3.

(2) For $A, B \in M_{n}(\mathbb{C})$ and $f(x)=\frac{x+1}{2}$, we define an inner product on $M_{n}(\mathbb{C})$ by

$$
\langle A, B\rangle=\operatorname{Tr}\left[A_{0}^{*} m_{f}\left(L_{\rho}, R_{\rho}\right) B_{0}\right]
$$

By Schwarz's inequality we have

$$
|\langle A, B\rangle|^{2} \leq\langle A, A\rangle\langle B, B\rangle
$$

Then we have

$$
\begin{aligned}
|\langle A, B\rangle|^{2} & =\left|\operatorname{Tr}\left[A_{0}^{*} \frac{L_{\rho}+R_{\rho}}{2} B_{0}\right]\right|^{2} \\
& =\left|\frac{1}{2} \operatorname{Tr}\left[A_{0}^{*} \rho B_{0}\right]+\frac{1}{2} \operatorname{Tr}\left[A_{0}^{*} B_{0} \rho\right]\right|^{2}
\end{aligned}
$$




$$
\begin{aligned}
& =\left|\frac{1}{2} \operatorname{Tr}\left[\rho B_{0} A_{0}^{*}\right]+\frac{1}{2} \operatorname{Tr}\left[\rho A_{0}^{*} B_{0}\right]\right|^{2} \\
& =\frac{1}{4}\left|\operatorname{Tr}\left[\rho\left\{A_{0}^{*}, B_{0}\right\}\right]\right|^{2}
\end{aligned}
$$

and

$$
\begin{aligned}
\langle A, A\rangle & =\operatorname{Tr}\left[A_{0}^{*} \frac{L_{\rho}+R_{\rho}}{2} A_{0}\right] \\
& =\frac{1}{2} \operatorname{Tr}\left[A_{0}^{*} \rho A_{0}\right]+\frac{1}{2} \operatorname{Tr}\left[A_{0}^{*} A_{0} \rho\right] \\
& =\frac{1}{2} \operatorname{Tr}\left[\rho A_{0} A_{0}^{*}\right]+\frac{1}{2} \operatorname{Tr}\left[\rho A_{0}^{*} A_{0}\right] \\
& =\frac{1}{2}\left|\operatorname{Var}_{\rho}^{0}\right|(A) .
\end{aligned}
$$

Then

$$
\left|\operatorname{Tr}\left[\rho\left\{A_{0}^{*}, B_{0}\right\}\right]\right|^{2} \leq\left|\operatorname{Var}_{\rho}^{0}\right|(A) \cdot\left|\operatorname{Var}_{\rho}^{0}\right|(B) .
$$

By taking $A^{*}$ in place of $A$

$$
\left|\operatorname{Tr}\left[\rho\left\{A_{0}, B_{0}\right\}\right]\right|^{2} \leq\left|\operatorname{Var}_{\rho}^{0}\right|\left(A^{*}\right) \cdot\left|\operatorname{Var}_{\rho}^{0}\right|(B) .
$$

Since $\left|\operatorname{Var}_{\rho}^{0}\right|(A)=\left|\operatorname{Var}_{\rho}^{0}\right|\left(A^{*}\right)$, we have the result.

\section{Remark}

Dou-Du's result (3) For $A, B \in M_{n}(\mathbb{C})$ and $\rho \in M_{n,+1}(\mathbb{C})$,

$$
\left|\operatorname{Var}_{\rho}^{0}\right|(A) \cdot\left|\operatorname{Var}_{\rho}^{0}\right|(B) \geq \frac{1}{4}\left|\operatorname{Tr}\left[\rho[A, B]^{0}\right]\right|^{2}+\frac{1}{4}\left|\operatorname{Tr}\left[\rho\left\{A_{0}, B_{0}\right\}^{0}\right]\right|^{2} .
$$

Corollary 3 For $A, B \in M_{n}(\mathbb{C})$ and $\rho \in M_{n,+1}(\mathbb{C})$,

$$
\left|\operatorname{Var}_{\rho}^{0}\right|(A) \cdot\left|\operatorname{Var}_{\rho}^{0}\right|(B) \geq\left|U_{\rho}\right|(A) \cdot\left|U_{\rho}\right|(B) \geq \frac{1}{4}|\operatorname{Tr}[\rho[A, B]]|^{2} .
$$

We cannot compare the RHS of (6) with the RHS of (7). In fact, let

$$
\rho=\left(\begin{array}{cc}
\frac{3}{4} & 0 \\
0 & \frac{1}{4}
\end{array}\right), \quad A=\left(\begin{array}{cc}
i & 1 \\
-1 & i
\end{array}\right), \quad B=\left(\begin{array}{ll}
0 & 1 \\
2 & 0
\end{array}\right)
$$

Since the RHS of $(6)=0$ and the RHS of $(7)=\frac{9}{16}$, we have the RHS of $(6)<$ the RHS of (7). On the other hand let

$$
\rho=\left(\begin{array}{cc}
\frac{3}{4} & 0 \\
0 & \frac{1}{4}
\end{array}\right), \quad A=\left(\begin{array}{cc}
i & 1 \\
-1 & i
\end{array}\right), \quad B=\left(\begin{array}{cc}
0 & 1 \\
-1 & 0
\end{array}\right) .
$$

Since the RHS of (6) = 1 and the RHS of $(7)=0$, we have the RHS of $(6)>$ the RHS of (7). 


\section{Conclusion}

The results (1), (4) of Dou-Du are given as corollaries of Theorem 3. Result (2) is proved by Schwarz's inequality. Also is shown, that (3) cannot be compared with our result.

\section{Competing interests}

The authors declare that they have no competing interests.

\section{Authors' contributions}

This work was carried out in collaboration between all authors. Example 2 and the comparison between (6) and (7) were given by KS. With the exception of them, the proofs of all results were given by KY. All authors have contributed to, checked, and approved the manuscript.

\section{Acknowledgements}

The first author (KY) was partially supported by JSPS KAKENHI Grant Number 26400119.

Received: 29 September 2015 Accepted: 13 November 2015 Published online: 03 December 2015

\section{References}

1. Dou, YN, Du, HK: Generalizations of the Heisenberg and Schrödinger uncertainty relations. J. Math. Phys. 54, 103508 (2013)

2. Dou, YN, Du, HK: Note on the Wigner-Yanase-Dyson skew information. Int. J. Theor. Phys. 53, $952-958$ (2014)

3. Furuichi, S, Yanagi, K: Schrödinger uncertainty relation, Wigner-Yanase-Dyson skew information and metric adjusted correlation measure. J. Math. Anal. Appl. 388, 1147-1156 (2012)

4. Lieb, EH: Convex trace functions and the Wigner-Yanase-Dyson conjecture. Adv. Math. 11, 267-288 (1973)

5. Luo, S, Zhang, Q: On skew information. IEEE Trans. Inf. Theory 50, 1778-1782 (2004); Correction to 'On skew information.' IEEE Trans. Inf. Theory 51, 4432 (2005)

6. Kosaki, H: Matrix trace inequality related to uncertainty principle. Int. J. Math. 16, 629-646 (2005)

7. Yanagi, K, Furuichi, S, Kuriyama, K: A generalized skew information and uncertainty relation. IEEE Trans. Inf. Theory 51, 4401-4404 (2005)

8. Hansen, F: Metric adjusted skew information. Proc. Natl. Acad. Sci. USA 105, 9909-9916 (2008)

9. Yanagi, K: Metric adjusted skew information and uncertainty relation. J. Math. Anal. Appl. 380, 888-892 (2011)

10. Yanagi, K, Furuichi, S, Kuriyama, K: Uncertainty relations for generalized metric adjusted skew information and generalized metric adjusted correlation measure. J. Uncertain. Anal. Appl. 1, 1-14 (2013)

11. Gibilisco, P, Hansen, F, Isola, T: On a correspondence between regular and nonregular operator monotone functions. Linear Algebra Appl. 430, 2225-2232 (2009)

12. Hiai, F, Petz, D: Convexity of qusi-entropy type functions: Lieb's and Ando's convexity theorems revisited. J. Math. Phys. $54,062201(2013)$

\section{Submit your manuscript to a SpringerOpen ${ }^{\ominus}$ journal and benefit from:}

- Convenient online submission

Rigorous peer review

- Immediate publication on acceptance

- Open access: articles freely available online

- High visibility within the field

- Retaining the copyright to your article 